\title{
Clot Histopathology in Ischemic Stroke with Infective Endocarditis
}

\author{
Sonu Bhaskar (D), Jawad Saab, Cecilia Cappelen-Smith, Murray Killingsworth, \\ Xiao Juan Wu, Andrew Cheung, Nathan Manning, Patrick Aouad, \\ Alan McDougall, Suzanne Hodgkinson, Dennis Cordato
}

\begin{abstract}
Background: Endovascular thrombectomy (EVT) has shown efficacy in acute ischemic stroke (AIS) patients with infective endocarditis (IE). The possibility to undertake advanced histopathological clot analysis following EVT offers a new avenue to establish the etiological basis of the stroke - which is often labelled "cryptogenic." In this paper, we present our findings from four consecutive patients with IE who underwent EVT following an AIS at our tertiary referral comprehensive stroke centre. Methods: Comprehensive histopathological analysis of clot retrieved after EVT, including morphology, was undertaken. Results: The consistent observation was the presence of dense paucicellular fibrinoid material mixed/interspersed with clusters of bacterial cocci. This clot morphology may be specific to septic embolus due to IE unlike incidental bacteraemia and could possibly explain the refractoriness of such clots to systemic thrombolysis. Conclusion: Detailed morphological and histopathological analysis of EVT-retrieved clots including Gram staining can assist in etiological classification of the clot. Understanding the composition of the clot may be of clinical value in early diagnostics and mapping treatment planning in IE.
\end{abstract}

RÉSUMÉ: Recourir à l'histopathologie des caillots de patients victimes d'accidents ischémiques et atteints d'endocardite infectieuse. Contexte: La thrombectomie endovasculaire (TEV) s'est révélée efficace dans le cas de patients aux prises avec une endocardite infectieuse qui ont été victimes d'accidents ischémiques cérébraux aigus. La possibilité de procéder à des analyses histopathologiques avancées de leurs caillots à la suite d'une TEV offre désormais une avenue inédite permettant d'établir les bases étiologiques d'un AVC, avenue souvent qualifiée de «cryptogénique ». Dans cet article, nous voulons présenter les résultats que nous avons obtenus auprès de quatre patients qui étaient atteints d'endocardite infectieuse et qui avaient subi une TEV à la suite d'un accident ischémique cérébral aigu. Fait à souligner, ces patients ont été vus un à la suite de l'autre après avoir été adressés à notre centre tertiaire complet de prise en charge des AVC (tertiary referral comprehensive stroke centre). Méthodes: Nous avons procédé à une analyse histopathologique exhaustive de caillots ayant été récupérés consécutivement à une TEV, notamment leur aspect morphologique. Résultats: De façon régulière, nous avons pu observer la présence de substance paucicellulaire fibrinoïde dense mélangée ou alternant avec des bactéries de forme sphérique regroupées en grappes. À la différence des bactériémies indirectes, il se pourrait que cette caractéristique morphologique se rapporte spécifiquement à une embolie septique attribuable en retour à une endocardite infectieuse. Cela pourrait expliquer éventuellement la réaction réfractaire de tels caillots à une thrombolyse effectuée systématiquement. Conclusion: Il est donc possible, si l'on veut procéder à une classification étiologique de caillots, de bénéficier d'analyses morphologiques et histopathologiques de caillots récupérés à la suite d'une TEV, ce qui inclut le recours à la technique de coloration de Gram. Il se pourrait ainsi que la compréhension de la composition d'un caillot soit d'une utilité clinique lors de diagnostics précoces d'endocardite infectieuse et aussi dans le but de planifier et d'établir un traitement.

Keywords: Ischemic stroke, Infective endocarditis, Endovascular thrombectomy, Clot histopathology doi:10.1017/cjn.2019.8

Can J Neurol Sci. 2019; 46: 331-336

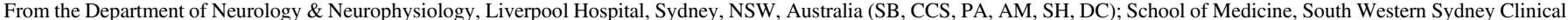

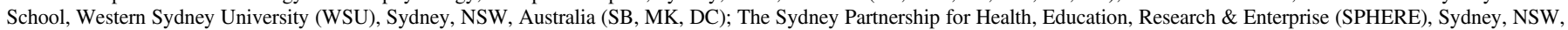

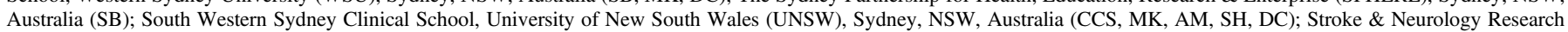

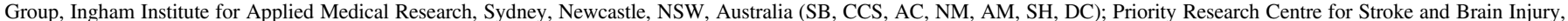

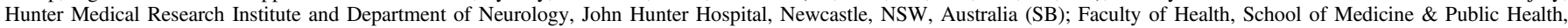

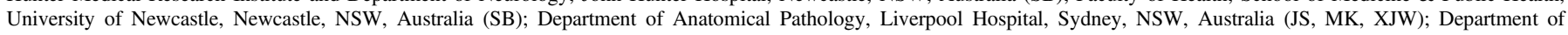

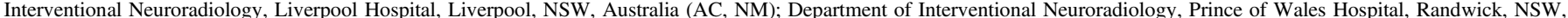

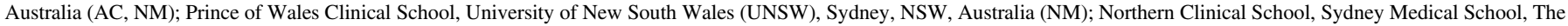
University of Sydney, Sydney, NSW, Australia (PA)

Received June 26, 2018. Final Revisions Submitted January 14, 2019. Date of Acceptance January 17, 2019.

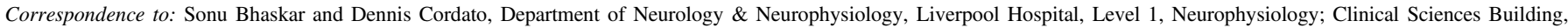

Elizabeth St, Liverpool Hospital, Sydney, NSW 2170, Australia. Emails: Sonu.Bhaskar@health.nsw.gov.au; Dennis.Cordato@health.nsw.gov.au 


\section{INTRODUCTION}

Septic embolus is a complication often seen in patients with infective endocarditis (IE). In this subgroup, 20\%-40\% patients are at increased risk of developing neurological complications such as acute ischemic stroke (AIS), intracerebral haemorrhage and mycotic aneurysm. ${ }^{1-3}$ Mortality rates as high as $56 \%$ have been reported in patients with stroke secondary to septic emboli. ${ }^{1,4}$

There is a lack of a defined pathway or consensus on the appropriate acute stage treatment strategy in patients with IE with large vessel occlusion (LVO). ${ }^{5}$ Systemic thrombolysis using intravenous tissue plasminogen activator (IV-tPA) may potentiate the risk of haemorrhage. ${ }^{1,5}$ To this end, endovascular thrombectomy (EVT) is emerging as a potentially efficacious and safer intervention strategy in this subgroup of patients. ${ }^{6,7}$ We recently reported a case study on the usefulness of EVT and clot histopathology in a patient with stroke secondary to IE. ${ }^{8}$ EVT also offers further benefit to pursue a thorough histopathologic analysis including routine Gram staining of EVT-retrieved clots, which may provide additional diagnostic/etiologic information in non-IE patients, ${ }^{9}$ and in those with suspected IE or prosthetic valvular heart disease. ${ }^{10}$ This perspective was unfortunately missing from the recently published consensus guidelines of thrombus analysis in AIS. ${ }^{11}$ In this paper, we present case series of four consecutive IE patients who received EVT at our comprehensive stroke centre (CSC). EVT-retrieved clots were sent for comprehensive histopathological and morphological analysis. We present our findings with emphasis on clot morphology, which may offer a unique window for better diagnostic characterisation and effective secondary stroke treatment.

\section{Materials AND Methods}

AIS patients with LVO aged $>18$ years admitted to our institution over a 12-month period (January 2017-January 2018) with IE who received EVT, which constituted 3.2\% (4/126) of EVT-treated patients, were included in this study. All patients underwent diagnostic scanning using routine AIS imaging protocol comprising of non-contrast computed tomography (NCCT), spiral CT angiography (CTA) from the aortic arch to circle of Willis and neuro CT perfusion (CTP). Clinical diagnosis of IE was confirmed using the Duke diagnostic criteria following routine diagnostic work-up including blood cultures, haematological analysis and transesophageal echocardiography (TEE). All patients underwent EVT using aspiration or combined stent retriever and aspiration technique under general anaesthesia. Ethics approval for the study was obtained from the South-Western Sydney Local Health District Human Research Ethics Committee.

\section{Liverpool Clot Histopathological Examination Protocol}

Prior to this case series, there was no standardised histopathological assessment of the EVT-retrieved clots at our institution. It was found that retrospective staining was required for Gram stain (Case 2), and so a comprehensive histopathological clot analysis protocol was developed to routinely examine prospective EVT-retrieved clots (Cases 1, 3 and 4). All EVT-retrieved clots were fixed in formalin in adherence with normal septic handling procedures. Formalin-fixed clots were then reviewed for their colour, size and morphology. Routine histology using haematoxylin and eosin (H\&E) stain and additional special stains were performed. For staining, formalin-fixed, paraffin-embedded
(FFPE) tissue block specimens were prepared. The special stain protocol included Gram staining (to distinguish gram-positive and gram-negative bacteria), the Periodic Acid Schiff for Fungus Stain Kit (PASF), Gomori's Methenamine-Silver Nitrate (GMS) stain (for screening fungal organisms), Masson Trichrome (for detection of collagen fibres in tissue sections), Von Kossa (for histologic visualisation of calcium deposits in tissue specimens), Verhoeff-Van Gieson elastic staining (EVG) (for detecting elastic fibres) and Perls' Prussian Blue staining (for histological visualisation of hemosiderin/iron).

\section{Results}

\section{Case Series}

\section{Case Study 1}

An 80-year-old man was presented with septic shock to a primary stroke centre, with a systolic blood pressure (SBP) of $70 \mathrm{mmHg}$ and sudden onset of right-sided hemiplegia and dysphagia. His past medical history included IE a year ago, mitral and atrial bioprosthetic valve replacements (M/AVR) and paroxysmal atrial fibrillation. TEE demonstrated vegetation on the anterior cusp of the transcatheter aortic valve implantation (TAVI) prostheses as well as an echo dense lesion on the atrial side of the A2 scallop of the mitral valve. The patient was in sinus rhythm during his subsequent transfer and admission to our CSC. He was febrile (temperature of $38.3^{\circ} \mathrm{C}$ ) and had received empirical treatment for recurrent IE with Flucloxacillin, Vancomycin and Gentamicin. National Institute of Health Stroke Scale (NIHSS) at presentation was 16 and Glasgow Coma Scale (GCS) was 11. Baseline NCCT showed hyperdense left M1 segment of the middle cerebral artery (MCA) artery with loss of grey-white matter differentiation in the left insular region consistent with an acute cerebral infarction. CTA showed occlusion of the left internal carotid artery (ICA) extending to the M1 segment of the MCA. CTP showed recent left MCA territorial infarction with large surrounding penumbra as demonstrated by decreased cerebral blood flow (CBF), decreased cerebral blood volume (CBV), prolonged mean transit time (MTT) and prolonged transient time to peak (TTP) in left MCA territory. Due to haemodynamic instability, the patient was presented to our CSC after a considerable delay. Groin access was established at $5 \mathrm{~h}$ 35 min following the symptom onset. EVT achieved good reperfusion with a modified thrombolysis in cerebral infarction (mTICI) score of $2 \mathrm{~b}$ at $7 \mathrm{~h} 45 \mathrm{~min}$ after the stroke onset. However, prerolandic cortical branch occlusion and proximal A1 occlusion of the anterior cerebral artery (ACA) persisted. Moderate sized area of infarction involving the left frontal and temporal lobes was observed on follow-up imaging following EVT. NIHSS at $24 \mathrm{~h}$ was 21.

The septic clot retrieved after EVT from the left ICA and M1 was sent for histopathology and Gram staining. The specimen consisted of three tan to dark brown fragments, with size varying from 2 to $28 \mathrm{~mm}$ in length and $2 \mathrm{~mm}$ in width. There were two morphologically distinct areas within the clot. The first, which occupied a quarter of the specimen, showed mostly acellular densely hyalinised fibrinoid material with essentially no blood and a few scattered neutrophils. Gram staining showed the presence of colonies of Gram-positive cocci in clusters in this material. The other three quarters of the specimen showed a fresh thrombus composed of blood and bands of fibrin mixed with 


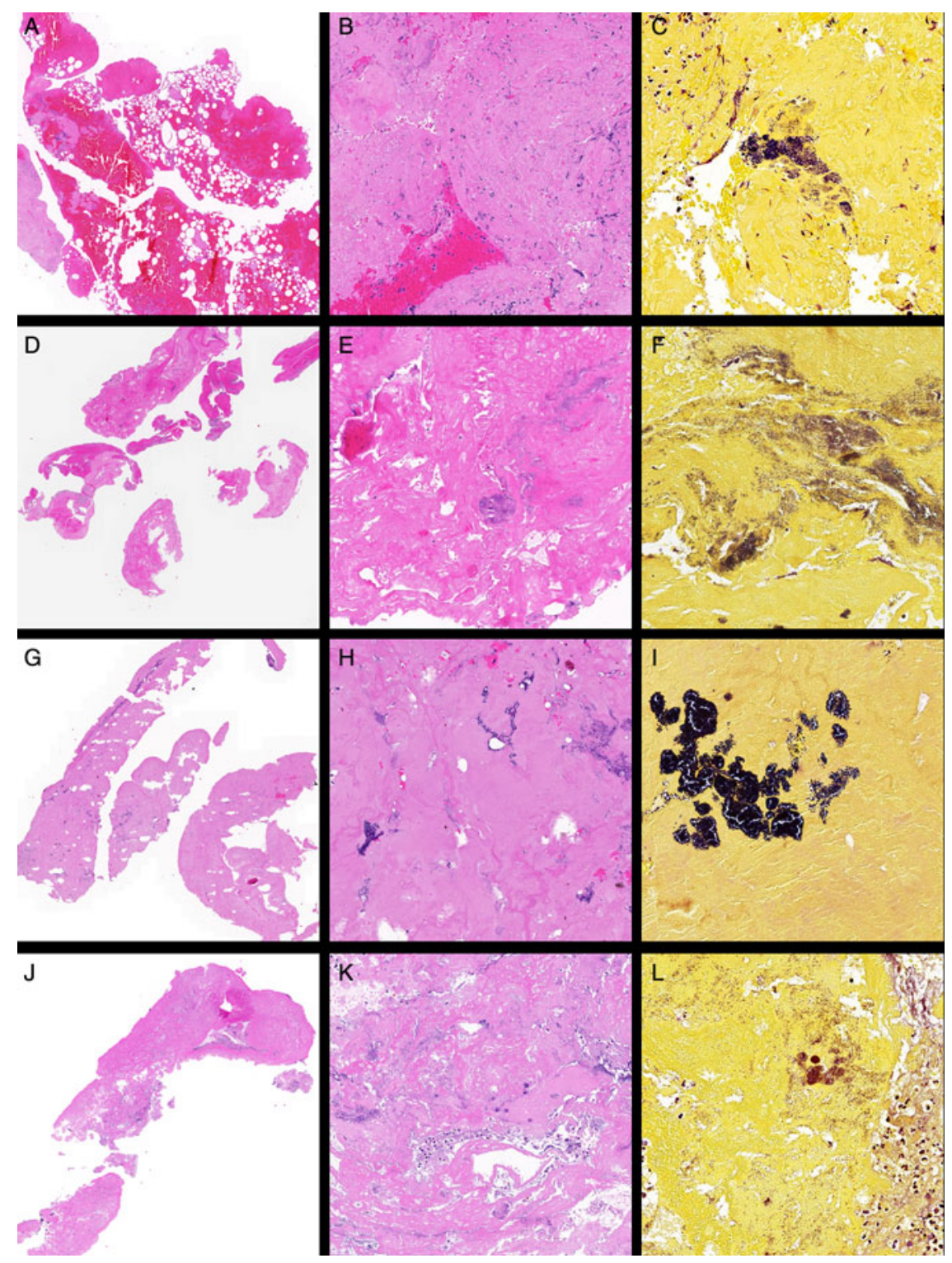

Figure 1: Representative histopathological images of the clots retrieved after EVT from patients with IE. Case 1: (A) Low-power view of fibrin-rich thromboembolus (left) with attached blood-rich thrombus (right) (H\&E stain, $\times 2),(B)$ high-power view of paucicellular fibrin-rich thromboembolus $(H \& E$ stain, $\times 20)$ and $(C)$ Gram stain showing colonies of Gram-positive cocci $(\times 40)$. Case 2: (D) Low-power view $(H \& E, \times 2),(E)$ high-power view of paucicellular fibrin-rich thromboembolus showing suspected colonies of bacteria $(H \& E$ stain, $\times 20)$ and $(F)$ Gram stain $(\times 40)$. Case 3: $(G)$ Low-power view of fibrin-rich thromboembolus $(H \& E, \times 2),(H)$ high-power view of paucicellular fibrin-rich thromboembolus showing colonies of bacteria $(H \& E$ stain, $\times 20)$ and (I) Gram stain $(\times 40)$. Case 4: (J) Low-power view of fibrin-rich thromboembolus $(H \& E, \times 2),(K)$ high-power view $(H \& E$ stain, $\times 20)$ and $(L)$ Gram stain $(\times 40)$.

variably sized empty vacuoles, presumed to be gas introduced at the time of the EVT procedure. The thin wispy bands of fibrin were associated with a few neutrophils and eosinophils, which tended to aggregate around the fibrin bands. No lymphocytes or cholesterol clefts were present. All other special stains were negative. Overall, these findings were consistent with a vegetation embolising from the heart in a background of IE and an associated fresh thrombus. The images of histopathological analysis for this case are shown in Figure 1(A)-(C).

\section{Case Study 2}

This case, of a 36-year-old man with a previous bioprosthetic aortic valve replacement (AVR) who developed an acute onset left hemiparesis whilst exercising, has previously been discussed in our recently published report. ${ }^{8}$ He was presented to our hospital following an episode of collapse and a baseline NIHSS of 16. CTA of the neck and circle of Willis revealed a right M1 occlusion. Patient received systemic thrombolysis and underwent EVT. EVT with aspiration achieved excellent reperfusion (mTICI 


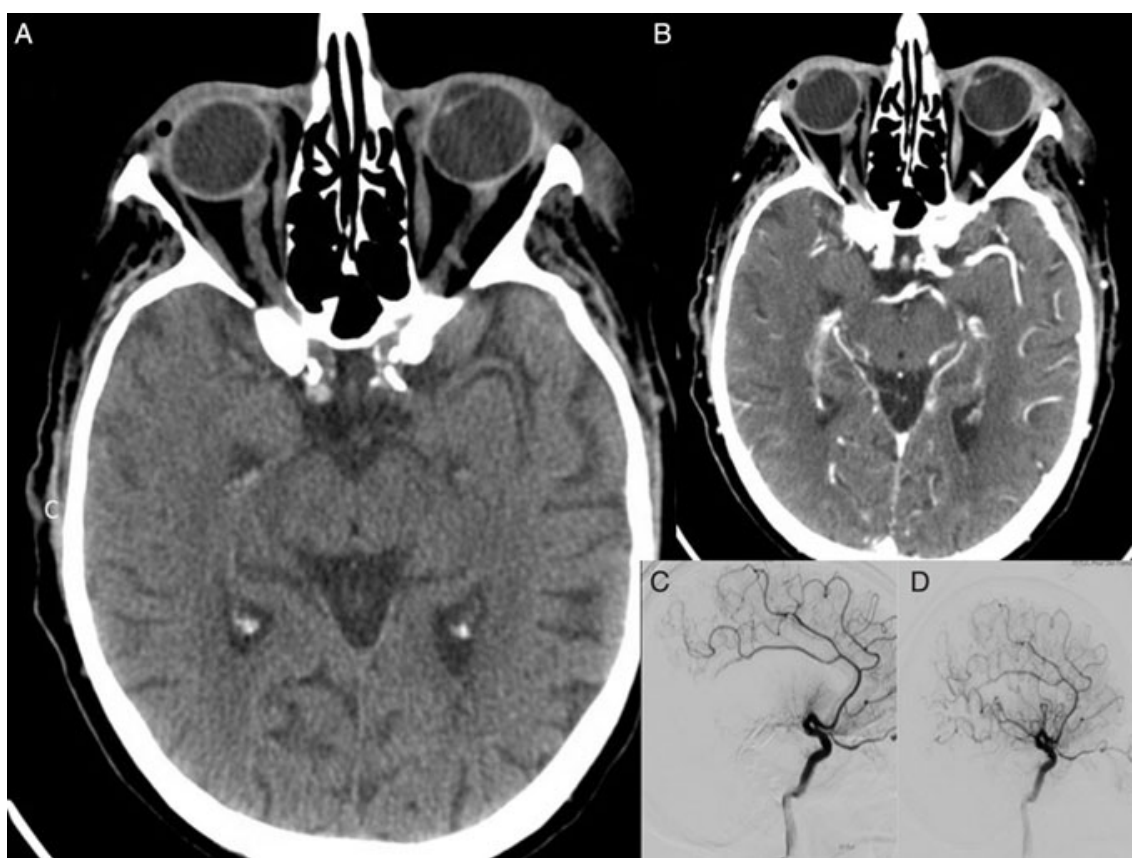

Figure 2: Pre- and post-EVT imaging for Case Study 3. Baseline NCCT showed hyperdense distal right M1 segment in the right MCA territory consistent with the evolving infarction (A). CT angiogram of the circle of Willis revealed abrupt cut-off at the distal M1 segment of the right MCA (B). Cerebral DSA confirmed mid-right M1 occlusion (C). Patient received EVT using single pass with Solitaire $4 \times 20 \mathrm{~mm}$ and aspiration via a Sofia Plus $6 F$. Following EVT, patient showed excellent angiographic reperfusion with mTICI score of $3(D)$. The follow-up, post-EVT, $C T$ scan showed acute infarction in the right frontal temporal cortex and external capsule. Subacute infarction in the right occipital cortex was also noted.

score 3$)$ at 3 h 30 min post-symptom onset. Routine histology using H\&E stains showed a fibrin-rich thromboembolus with acute inflammatory cells. At this time, Gram stain was not routinely used for examination of EVT clots. Extensive investigation in hospital did not establish a cardiac source of embolism. His recovery profile was excellent with NIHSS at $24 \mathrm{~h}$ of 2 . He was discharged home after 5 days with modified Rankin score (mRS) of 1 on daily regimen of metoprolol $(25 \mathrm{mg})$ and aspirin (100 mg). The patient developed polyarthralgias without evident fever 3 weeks post-discharge. A large aortic valve vegetation was confirmed on repeat echocardiography. Routine histology using H\&E stains showed fibrin-rich thromboembolus with acute inflammatory cells, and retrospective Gram staining of the initial clot confirmed septic embolism (see Figure 1(D)-(F)). Gram stain showed colonies of Gram-positive cocci. Follow-up MRI showed a right MCA territory subacute infarct with mass effect and midline shift.

\section{Case Study 3}

A 91-year-old man was presented with dense left-sided hemiparesis and slurred speech. He had a past history of ischemic heart disease (IHD) and aortic bioprosthetic valve replacement for aortic stenosis, clinically recurrent IE (previously treated with complete course of intravenous antibiotics for 6 weeks), hypertension, dyslipidaemia, severe mitral annular calcification with mild-to-moderate mitral stenosis and right upper lung lobe and colonic adenocarcinoma (both resected more than 5 years ago). Echocardiography revealed prosthetic aortic valve thrombosis suggestive of the valve vegetation. Patient was clinically diagnosed as having IE in accordance with the Duke criteria. NIHSS at admission was 19 . Baseline NCCT imaging showed high density in the right M1 segment of the MCA without evidence of established infarction. CTA confirmed abrupt cut-off with nonfilling of the distal right M1 segment of the MCA (see Figure 2). Moderate-grade calcific stenosis was observed on the proximal ICA bilaterally and high-grade stenosis was also seen on the left intracranial portion of the vertebral artery. Patient received tPA thrombolysis followed by EVT. EVT achieved excellent reperfusion with mTICI 3 score at 3 h 30 min after symptom onset (Figure 2(D)). Neurological improvement was noted following the procedure. The images pertaining to this case are presented in Figure 1. Magnetic resonance imaging (MRI) showed a subacute right MCA territory infarct with mass effect and mild midline shift. Follow-up MR angiography showed no evidence of mycotic aneurysm. Blood cultures grew Enterococcus faecalis confirming recurrence of IE. The patient was managed with ampicillin, gentamycin and amoxicillin. His 24-h NIHSS was 11 . He was discharged from a hospital with an mRS score of 4

Histopathological analysis was performed on the retrieved clot specimen (Figure 1(G)-(I)). The clot consisted of six fragments of $1-10 \mathrm{~mm}$ in length. Microscopic examination revealed small fragments of embolus comprising of blood, fibrin and aggregates of neutrophils. Colonies of both Gram-positive and Gram-negative cocci, possibly from cardiac valve consistent with IE, were observed on Gram stain in the fibrin-rich areas of the clot (Figure 1(I)). 


\section{Case Study 4}

A 71-year-old female with history of coronary artery bypass graft $(\mathrm{CABG})$ for dual vessel disease and metallic aortic valve replacement (AVR), paroxysmal atrial fibrillation (on warfarin regimen), hypertension and hypercholesterolemia presented with left-sided upper limb weakness on a background of methicillin-resistant Staphylococcus aureus (MRSA) sepsis. Echocardiography revealed the presence of vegetation on the metallic aortic valve. Her baseline NIHSS was 26.

Baseline NCCT revealed loss of grey/white matter differentiation in the right MCA territory consistent with a right MCA stroke. CTA confirmed complete occlusion of the right ICA extending from its proximal portion (approximately $15 \mathrm{~mm}$ distal to its origin) to its termination. Minimal flow was also observed in the right M1/proximal M2 with the absence of distal flow. There was crossover flow from the left ICA via the anterior communicating artery into the right ACA. CTP demonstrated an established ischemic core and large penumbra in the right MCA territory. EVT with combined stent retriever and aspiration achieved excellent mTICI 3 reperfusion at $6 \mathrm{~h} 10 \mathrm{~min}$ after the stroke onset. NIHSS score at $24 \mathrm{~h}$ was 12.

Clot retrieved from the right ICA and MCA was sent for histopathological analysis. The clot consisted of two fragments, approximately of 1-mm width each, and 5 and $30 \mathrm{~mm}$ length, respectively. Macroscopic examination of the whole clot showed pinkish-red soft tissue appearance. Microscopy showed dense fibrinoid material mixed with clusters of neutrophils with no evidence of fresh blood (Figure 1(J) and (K)). Routine histology showed numerous colonies of bacteria scattered throughout the clot. Gram staining demonstrated the presence of numerous coccal organisms including some round-shaped Gram-positive bacteria, confirming the earlier positive blood cultures indicative of $S$. aureus (Figure 1(L)). No lymphocytes or cholesterol clefts were observed. However, Von Kossa and Perl's Prussian Blue staining revealed the presence of small nodule of calcification and scattered granules of hemosiderin, respectively. EVG staining for the elastic fibres was negative. Interestingly, collagen-rich soft tissue was seen within the fibrinoid material (on Masson Trichrome staining), presumably small fragments of the vessel wall.

\section{Discussion}

EVT, with or without systemic thrombolysis, has revolutionised stroke treatment and is being increasingly adopted as the standard of acute stroke treatment. ${ }^{12}$ However, this highly specialised intervention can be only offered to much selected group of patients with favourable pre-treatment CT imaging profiles. These too must be obtained within a constrained time window of $4.5 \mathrm{~h}$ since stroke onset for intravenous thrombolysis and $6 \mathrm{~h}$ and up to $24 \mathrm{~h}$ for EVT in selected patients. ${ }^{13}$ Therefore, only a few patients with stroke and IE are likely to receive this intervention, but this will change as time progresses.

Our case series is therefore exceedingly rare. ${ }^{14} \mathrm{~A}$ recent study on histopathological and bacteriological analyses of EVT-retrieved IE clots by Hernandez-Fernandez et al. reported an increased proportion of septic emboli diagnosis. ${ }^{14}$ Stroke patients with IE are most likely to have very poor prognosis. In the milieu of EVT, we now have a rare opportunity to study the clot profiles and see its value in management of stroke patients. In our series of four cases, the novel finding was the unique brain clot morphology of paucicellular fibrin bands around vegetation which may be commonly seen by cardiovascular pathologists, but have not been reported in extracted brain clots - to the best of our knowledge. The composition of these clots is different from nonseptic embolic clots. ${ }^{14,15}$ Stroke patients with IE often respond very poorly to reperfusion intervention including IV-tPA and till date we have very limited understanding of why these patients respond as they do. In our case series, patients responded favourably to EVT.

This study on a case series presents an account of the value of clot analysis and EVT in patients with suspected IE and valvular heart disease and artificial or bioprosthetic valve replacements. It also presents a detailed characterisation of the EVT-retrieved clots in these patients using comprehensive histopathological and morphological analysis.

Histopathological and Gram staining may have a value in routine pathology work-up to confirm clinical diagnoses and as an adjunct decision-making tool for prompt treatment planning in acute stroke settings. Gram staining of the clots was able to confirm the diagnosis of septic embolism due to IE and classify the clot as a septic embolus. A consistent observation in our case series was the presence of dense paucicellular fibrinoid material mixed with clusters of bacterial cocci. This morphology is remarkably unique to septic emboli and may be useful in differential diagnoses of IE over incidental bacteraemia. However, differential features for IE and incidental bacteraemia need further research. To our knowledge, this is the first study describing the presence of this characteristic morphology using detailed histopathological and morphological analysis of EVT-retrieved clots including Gram staining, which may be of value in early diagnostics and treatment planning in IE.

The interspersion of red blood cells (RBCs) within bands of fibrin is probably due to in situ formation of fresh clot around the embolus. In the milieu of septic embolisation, IV-tPA has been reported to be associated with increased risk of bleeding and poor lytic efficacy - hence increased probability of poor angiographic and clinical outcomes. ${ }^{5,16}$ Consistent with the predominance of RBCs interspersed within fibrin bands in our retrieved clots, we found prominent early imaging signs such as the hyperdense middle cerebral artery (HDMCA) sign on NCCT in all four cases. Other studies have also reported a correlation between these early imaging signs with the density of RBCs in the retrieved thrombi of non-IE-related cases. ${ }^{15,17-20}$

From a therapeutic perspective, it is advisable to initiate aggressive antibiotic therapy as early as possible in patients suspected with IE or with associated risk factors. This may potentially thwart further septic embolisation and cerebral infarction. ${ }^{5}$ Management of ischemic stroke patients with LVO and IE is clinically challenging due to lack of specific guidelines. It has been observed that the risk of embolisation may be higher in patients with large $(>10 \mathrm{~mm})$, left-sided mitral valve vegetation. ${ }^{5}$

Given that the diagnosis of IE is often delayed, and in some cases missed, there is an inherently increased risk of morbidity and mortality for the patient. Detailed clot histopathology including Gram staining should be undertaken in patients at heightened risks of IE such as those with native or prosthetic valvular heart disease. Clot morphology may be useful in distinguishing septic emboli from incidental bacteraemia. We acknowledge that septic emboli are not necessarily synonymous with IE. The clot analyses 
in all our four cases supported the clinical diagnoses of IE, and the emboli in these cases were pathological vegetations, thereby fulfilling the Duke criteria.

\section{LiMITATIONS}

The findings of this report should be appreciated within the context of this study population. Methodological limitations of this study include small sample size and lack of control cases, such as those with incidental bacteraemia, for comparison. Since the clot analysis confirmed clinical diagnoses of IE in this case series, the clot analysis including Gram staining did not necessarily change the treatment course. However, it is still useful in detailed characterisation and classification of the EVT-retrieved clots.

\section{Conclusions}

Understanding the composition and morphology of clots may offer a robust explanation on why patients with IE respond poorly to thrombolysis and carry increased risk of poor outcomes. We hypothesise, based on current findings, that variation in clot composition revealed by comprehensive histopathological examination may be useful in etiological characterisation.

\section{ACKNOWLEDGEMENTS}

We would like to thank our staff members from the Department of Pathology at Liverpool Hospital for their feedback and ongoing discussions around clot analysis.

\section{Disclosures}

The authors have no conflicts of interest to declare.

\section{Statement of Authorship}

SB, JS, MK and DC conceptualised the study. SB, JS, XJW and MK developed the histopathological protocol. SB wrote the first draft of the manuscript. All authors contributed to the revision, overall discussion and conduct of the study.

\section{REFERENCES}

1. Walker KA, Sampson JB, Skalabrin EJ, Majersik JJ. Clinical characteristics and thrombolytic outcomes of infective endocarditis-associated stroke. Neurohospitalist. 2012;2(3):87-91.

2. Hart RG, Foster JW, Luther MF, Kanter MC. Stroke in infective endocarditis. Stroke. 1990;21(5):695-700.

3. Morris NA, Matiello M, Lyons JL, Samuels MA. Neurologic complications in infective endocarditis: identification, management, and impact on cardiac surgery. Neurohospitalist. 2014; 4(4):213-22.
4. Ruttmann E, Willeit J, Ulmer H, et al. Neurological outcome of septic cardioembolic stroke after infective endocarditis. Stroke. 2006;37(8):2094-9.

5. Kim JM, Jeon JS, Kim YW, Kang DH, Hwang YH, Kim YS. Forced arterial suction thrombectomy of septic embolic middle cerebral artery occlusion due to infective endocarditis: an illustrative case and review of the literature. Neurointervention. 2014;9(2):101-5.

6. Liang JJ, Bishu KG, Anavekar NS. Infective endocarditis complicated by acute ischemic stroke from septic embolus: successful solitaire FR thrombectomy. Cardiol Res. 2012;3(6):277-80.

7. Sveinsson O, Herrman L, Holmin S. Intra-arterial mechanical thrombectomy: an effective treatment for ischemic stroke caused by endocarditis. Case Rep Neurol. 2016;8(3):229-33.

8. Bhaskar S, Cordato D, Cappelen-Smith C, et al. Clarion call for histopathological clot analysis in "cryptogenic" ischemic stroke: implications for diagnosis and treatment. Ann Clin Transl Neurol. 2017;4(12):926-30.

9. Niesten JM, van der Schaaf IC, van Dam L, et al. Histopathologic composition of cerebral thrombi of acute stroke patients is correlated with stroke subtype and thrombus attenuation. PloS One. 2014;9(2):e88882.

10. Boeckh-Behrens T, Kleine JF, Zimmer C, et al. Thrombus histology suggests cardioembolic cause in cryptogenic stroke. Stroke. 2016;47(7):1864-71.

11. De Meyer SF, Andersson T, Baxter B, et al. Analyses of thrombi in acute ischemic stroke: a consensus statement on current knowledge and future directions. Int J Stroke: Off J Int Stroke Soc. 2017;12(6):606-14.

12. Bhaskar S, Stanwell P, Cordato D, Attia J, Levi C. Reperfusion therapy in acute ischemic stroke: dawn of a new era? BMC Neurol. 2018;18:8.

13. Nogueira RG, Jadhav AP, Haussen DC, et al. Thrombectomy 6 to 24 hours after stroke with a mismatch between deficit and infarct. N Engl J Med. 2017;378(1):11-21.

14. Hernandez-Fernandez F, Rojas-Bartolome L, Garcia-Garcia J, et al. Histopathological and bacteriological analysis of thrombus material extracted during mechanical thrombectomy in acute stroke patients. Cardiovasc Interv Radiol. 2017;40(12):1851-60.

15. Brinjikji W, Duffy S, Burrows A, et al. Correlation of imaging and histopathology of thrombi in acute ischemic stroke with etiology and outcome: a systematic review. J Neurointerv Surg. 2017; 9(6):529-34.

16. Brownlee WJ, Anderson NE, Barber PA. Intravenous thrombolysis is unsafe in stroke due to infective endocarditis. Intern Med J. 2014;44(2):195-7.

17. Kim SK, Yoon W, Kim TS, Kim HS, Heo TW, Park MS. Histologic analysis of retrieved clots in acute ischemic stroke: correlation with stroke etiology and gradient-echo MRI. AJNR Am J Neuroradiol. 2015;36(9):1756-62.

18. Niesten JM, van der Schaaf IC, van Dam L, et al. Histopathologic composition of cerebral thrombi of acute stroke patients is correlated with stroke subtype and thrombus attenuation. PloS One. 2014;9(2):e88882.

19. Qazi EM, Sohn SI, Mishra S, et al. Thrombus characteristics are related to collaterals and angioarchitecture in acute Stroke. Can J Neurol Sci. 2015;42(6):381-8.

20. Liebeskind DS, Sanossian N, Yong WH, et al. CT and MRI early vessel signs reflect clot composition in acute stroke. Stroke. 2011;42(5):1237-43. 